\title{
Particle simulation of diffusion with non uniform viscosity
}

\author{
S. Huberson, O. Le Maître, E. Rivoalen \\ Laboratoire de Mécanique, Université du Havre, 25, rue P. Lebon, BP540, 76053 Le Havre \\ Cedex, France e-mail hu@cher.univ-lehavre.fr
}

A lot of turbulence models are based on the turbulent viscosity concept. This results in the introduction of a non uniform diffusion process in the Navier Stokes equations. The aim of this work is to investigate the capability of some existing particle modelling to solve this kind of problem. Three different methods have been selected, the Strength Exchange Method, the Random Vortex Method and the Diffusion Velocity Method. All three methods have been tested on the problem of the turbulent transport of a passive scalar in a jet.

\section{Introduction}

The extension of particle methods to viscous flows in the last ten years has resulted in the emergence of many different diffusion simulation algorithms. The next step for particle methods is the extension to turbulent modelling. Although there is a possibility to derived new models taking advantages of the Lagrangian formulation, it seems that it will be eventually necessary to solve a more complex vorticity transport equation, including a non uniform diffusion process. The extension and validation of the existing diffusion methods to this kind of problem is the objective of the present work. Only grid free methods and 2D diffusion for a passive scalar have been considered. Although the actual vorticity transport equation contains additional terms according to the different modelization strategies ${ }^{10,1,12}$, we will restrict the study to the following convection-diffusion equation :

$$
\frac{\partial c}{\partial t}+\underline{\nabla} \cdot(\underline{\mathbf{U}} c)=\underline{\nabla} \cdot(\nu \underline{\nabla} c)
$$

\section{Numerical Models}

\subsection{The Particle approximation}

There is an impressive variety of the published methods. However it is possible to roughly classify them into three classes : the Random Vortex Model ${ }^{3,12}$ the Strength Exchange Method ${ }^{2,4,5,6,9}$ and the Diffusion Velocity Method ${ }^{5,7,11,12}$. Accordingly, three different methods have been tested. A short description of these methods is given hereafter.

Let $c^{o}(\underline{\mathbf{X}})$ be the particle approximation of $c^{o}(\underline{\mathbf{X}})=c(\underline{\mathbf{X}}, 0)$ :

$$
c_{\epsilon}^{o}(\underline{\mathbf{X}})=\sum_{i=1}^{N_{p}} C_{i}^{o} \xi_{\epsilon}\left(\underline{\mathbf{X}}-\underline{\mathbf{X}}_{i}\right) \approx c^{o}(\underline{\mathbf{X}})
$$


where $N_{p}$ is the total number of particles, $C_{i}^{o}$ are the initial weights, $\underline{\mathbf{X}}_{i}$ the locations of the particles, and $\xi_{\epsilon}$ a smooth function. An approximate solution $c_{\epsilon}(\underline{\mathbf{X}}, t)$ of $\mathrm{Eq}(1)$ is :

$$
c_{\epsilon}(\underline{\mathbf{X}}, t)=\sum_{i=1}^{N_{p}} C_{i}(t) \xi_{\epsilon}\left(\underline{\mathbf{X}}-\underline{\mathbf{X}}_{i}(t)\right)
$$

where $C_{i}$ and $\underline{\mathbf{x}}_{i}$ are solutions of the following differential equations :

$$
\begin{array}{r}
\frac{d \underline{\mathbf{X}}_{i}}{d t}=\underline{\mathbf{U}}_{\epsilon}\left(\underline{\mathbf{X}}_{i}\right)+\underline{\mathbf{U}}_{d}\left(\underline{\mathbf{X}}_{i}, \nu\right) \\
\frac{d C_{i}}{d t}=F_{i}\left(\underline{\mathbf{X}}_{j}, C_{j}, \nu\right)
\end{array}
$$

The choice of $\underline{\mathbf{U}}_{d}$ and $F_{i}$ characterises the method.

\subsection{Random Walk Model}

The RVM is based on the use of the Brownian motion to simulate the effect of diffusion. The convection diffusion equation has to be rewritten in order to obtain a suitable form for the diffusion operator :

$$
\begin{array}{r}
\frac{d c}{d t}+\underline{\nabla} \cdot(\underline{\mathbf{U}} c)=\nu \nabla^{2} c+\underline{\nabla} \nu \cdot \underline{\nabla} c \\
\frac{d c}{d t}+\underline{\nabla} \cdot[(\underline{\mathbf{U}}-\nabla \nu) c]=\nu \nabla^{2} c
\end{array}
$$

The resulting method is a constant weight particle model in which the particle pathes are governed by the following Langevin equation

$$
\left\{\begin{array}{l}
\underline{\mathbf{X}}_{i}(t+\delta t)=\int_{t}^{t+\delta t}\left(\underline{\mathbf{U}}+\underline{\mathbf{U}}_{\nu}\right) d \tau+\eta\left(\underline{\mathbf{X}}_{i}\right) \\
\underline{\mathbf{U}}_{\nu}=-\underline{\nabla} \nu \\
C_{i}(t+\delta t)=C_{i}(t)
\end{array}\right.
$$

where $\eta_{i}$ is a Gaussian random walk with variance : $4 \nu\left(\underline{\mathbf{X}}_{i}\right) \delta t$.

The viscosity gradient has been computed according to :

$$
\underline{\mathbf{U}}_{d}(\underline{\mathbf{X}})=-\underline{\nabla} \nu \approx-\sum_{j} \nu\left(\underline{\mathbf{X}}_{j}\right) \frac{\partial \xi_{\epsilon}\left(\underline{\mathbf{X}}-\underline{\mathbf{X}}_{j}\right)}{\partial \underline{\mathbf{X}}}
$$

\subsection{Strength Exchange Model}

This method consists in solving at every time step the diffusion equation :

$$
\frac{\partial c}{\partial t}=\nu \nabla \cdot(\underline{\nabla}(c))
$$


with a set of Dirac as an initial condition. Due to the linearity of this equation, the solution can be constructed as a sum of elementary solutions for the following problem :

$$
\begin{array}{r}
\frac{\partial c}{\partial t}=-\nu \Delta(c) \\
c(\underline{\mathbf{X}}, 0)=\delta(|\underline{\mathbf{X}}|)
\end{array}
$$

the solution $c$ of which is :

$$
c(\underline{\mathbf{X}}, t)=\frac{1}{4 \pi \nu t} \exp -\frac{\underline{\mathbf{X}}^{2}}{2 \pi \nu t}
$$

Using the particles yields the approximation :

$$
C_{i}(t+\delta t)=C_{i}(t)+\sum_{j}\left(C_{j}(t)-C_{i}(t)\right) \frac{1}{4 \pi \nu \delta t} \exp ^{-\frac{\left|\underline{\mathbf{X}}_{i}-\underline{\mathbf{X}}_{j}\right|^{2}}{2 \pi \nu \delta t}}
$$

In order to apply the SEM, Eq (1) has to be modified in :

$$
\frac{\partial c}{\partial t}+\underline{\nabla} \cdot[(\underline{\mathbf{U}}+\underline{\nabla} \nu) c]=\underline{\nabla} \cdot(\underline{\nabla}(\nu c))
$$

leading to the discrete solution :

$$
\begin{aligned}
C_{i}(t+\delta t 0) & =C_{i}(t)+\sum_{j}\left(\nu\left(\underline{\mathbf{X}}_{j}, t\right) C_{j}(t)-\nu\left(\underline{\mathbf{X}}_{i}, t\right) C_{i}(t)\right) \frac{1}{4 \pi \delta t} \exp ^{-\frac{\left|\underline{\mathbf{x}}_{i}-\underline{\mathbf{X}}_{j}\right|^{2}}{2 \pi \delta t}} \\
\underline{\mathbf{X}}_{i}(t+\delta t) & =\underline{\mathbf{X}}_{i}(t)+(\underline{\mathbf{U}}+\underline{\nabla} \nu) \delta t
\end{aligned}
$$

This is not the only way to discretise the convection diffusion equation and a wider class of model have been extensively studied in ${ }^{9}$. The main interest of this scheme is that it is based on the Green function of the diffusion operator. Thus it has been built according to a direct analysis of a diffusion process rather than according to a discretisation scheme. The major drawback is the accuracy of the method which is limited to second order.

\subsection{Diffusion Velocity Model}

The DVM yields a constant weight model in which the Fick law associated with the diffusion process has been used to express the equation in a divergence form :

$$
\begin{cases}\frac{\partial c}{\partial t}+\underline{\nabla} \cdot[\underline{\mathbf{U}} c+\underline{\mathbf{J}}(\underline{\mathbf{X}}, t)]=0 & \text { Conservation } \\ \underline{\mathbf{J}}(\underline{\mathbf{X}}, t)=-\nu \underline{\nabla} c & \text { Fick's law }\end{cases}
$$

denoting :

$$
\underline{\mathbf{U}}_{\nu}(\underline{\mathbf{X}}, t) c(\underline{\mathbf{X}}, t)=\underline{\mathbf{J}}(\underline{\mathbf{X}}, t)=-\nu(\underline{\mathbf{X}}) \cdot \underline{\nabla} c(\underline{\mathbf{X}}, t)
$$

This equation can be written in the form of a simple convection equation : 


$$
\frac{\partial c}{\partial t}=\underline{\nabla} \cdot\left(\left(\underline{\mathbf{U}}+\underline{\mathbf{U}}_{\nu}(\underline{\mathbf{X}}, t)\right) c\right)=0
$$

where :

$$
\underline{\mathbf{U}}_{\nu}(\underline{\mathbf{X}}, t)=-\nu(\underline{\mathbf{X}}) \cdot \frac{\underline{\nabla} c(\underline{\mathbf{X}}, t)}{c(\underline{\mathbf{X}}, t)} .
$$

From which a particle approximation is readily obtained :

$$
\begin{array}{r}
\frac{d \underline{\mathbf{X}}_{i}}{d t}=A\left(\underline{\mathbf{X}}_{i}(t)\right)+\underline{\mathbf{U}}\left(\underline{\mathbf{X}}_{i}\right) \\
\underline{\mathbf{U}}_{\nu}(\underline{\mathbf{X}})=-\nu(\underline{\mathbf{X}}) \cdot \frac{\sum_{j=1}^{n p} C_{j} \underline{\nabla} \xi\left(\underline{\mathbf{X}}-\underline{\mathbf{X}}_{j}\right)}{\sum_{j=1}^{n p} C_{j} \xi\left(\underline{\mathbf{X}}-\underline{\mathbf{X}}_{j}\right)}
\end{array}
$$

\section{$3 \quad$ External boundary conditions}

\subsection{Definition of the problem}

One of the advantages of the particle method is a natural account for external boundary conditions in the integral formulation used. However, the method is still confronted to the difficulty of discretizing an infinite domain with a finite number of particles. Several methods have been proposed to overcome this problem including an extension of the usual re-gridding procedures. Re-gridding technique has been found unavoidable in order to warranty a minimum accuracy in the case of highly sheared flows ${ }^{8}$. In the present work, it has not been used for two main reasons :

1. The aim of this work is to compare different discretisation schemes in the same conditions. The result would have been very difficult to analyse if a re-gridding technique was used since the location of the particles can be very different for the three methods. It is clear that this choice has resulted in a non-optimal form of the methods so that we do not claim that the conclusion of this work are definitive in any way.

2. The particle methods are now currently used to analyse the flow around propellers and wings and almost all these applications do not include any re-gridding procedure.

As a result, we expect our results to be of some interest for people using particle methods for complex flows simulation although some of the conclusions could be possibly wrong for those who are concentrating on the theoretical accuracy of the method.

Regarding the external boundary condition problem, the three methods are somewhat different since the domain covered by the RVM and DVM increased with time whereas the domain covered by the SEM particle is theoretically assume to be infinite. This condition is actually never satisfied and some practical correction procedures are proposed hereafter. Three different methods have been investigated :

1. Increase the Particles number

ESAIM: Proc., Vol. 7, 1999, $198-204$ 
2. Evaluate explicitly the out-flux

3. Modify the particles volume

Increasing the particle number is straightforward and only the last two method will be described hereafter.

\subsection{Explicit Evaluation of the out-flux}

For simplicity, only the constant viscosity case is considered. The particles contribution to Eq (10) can be split into two parts :

$$
C_{i}(t+\delta t)-C_{i}(t)=\sum_{j=1, I, j \neq i} C_{j}(t)\left|\mathcal{P}_{i}\right| \frac{1}{8(\pi \nu \delta t)^{3 / 2}} \exp \frac{\left(\underline{\mathbf{X}}_{i}-\underline{\mathbf{X}}_{j}\right)^{2}}{4 \nu \delta t}-\Phi_{i}
$$

where

$$
\Phi_{i}=\iiint_{\mathcal{P}_{i}} \iiint_{\mathbb{R}^{3}-\left\{\mathcal{P}_{i}\right\}} \frac{c(\underline{\mathbf{x}}, t)}{8(\pi \nu \delta t)^{3 / 2}} \exp \frac{\left(\underline{\mathbf{x}}-\underline{\mathbf{x}}^{\prime}\right)^{2}}{4 \nu \delta t} d \underline{\mathbf{x}^{\prime}} d \underline{\mathbf{x}}
$$

An exact evaluation of $\Phi_{i}$ is not possible since the shape of the particle is not known. However, the integral can be easily computed for the approximation of spherical particles with radius :

$$
\epsilon=\left(\frac{3}{4}\left|\mathcal{P}_{i}\right|\right)^{1 / 3}
$$

leading to :

$$
\Phi_{i, \epsilon}=\int_{o}^{2 \pi} \int_{o}^{\pi} \int_{o}^{\epsilon} \int_{o}^{2 \pi} \int_{o}^{\pi} \int_{\epsilon}^{\infty} \frac{c(\underline{\mathbf{x}}, t)}{8(\pi \nu \delta t)^{3 / 2}} \exp \frac{\left(\underline{\mathbf{x}}-\underline{\mathbf{x}}^{\prime}\right)^{2}}{4 \nu \delta t} \underline{\mathbf{d r}}^{\prime} \underline{\mathbf{d r}}
$$

Approximating again $\left.c\right|_{\mathcal{P}_{i}} \simeq C \delta_{o}$ yield :

$$
\Phi_{i, \epsilon}=-C_{i}\left[\frac{\epsilon}{\sqrt{\pi \nu \delta t}} \exp \left(-\frac{\epsilon^{2}}{4 \nu \delta t}+\operatorname{erfc}\left(\frac{\epsilon}{2 \sqrt{\nu \delta t}}\right)\right]\right.
$$

It is of some interest to point out that the flux $\Phi_{i}$ is a product of $C_{i}$ by a constant. If the first part of $\mathrm{Eq}(11)$ is neglected, the solution is a time exponential. Since the particles do not actually cover the infinite domain which is accounted for in this expression, the method can be expected to be dissipative. This has been actually observed in the computations. 


\subsection{Modify the particles volume}

For some Green Function $\mathcal{G}\left(\underline{\mathbf{x}}, \underline{\mathbf{x}}^{\prime}, t\right)$ one has :

$$
\iiint_{\mathbb{R}^{3}} \mathcal{G}\left(\underline{\mathbf{x}}_{o}, \underline{\mathbf{x}}^{\prime}, t\right) d \underline{\mathbf{x}}^{\prime}=1
$$

A discrete form of which is :

$$
\sum_{i=1, I} \mathcal{G}\left(\underline{\mathbf{x}}_{o}, \underline{\mathbf{x}}_{i}, t\right)\left|\mathcal{P}_{i}\right|=1
$$

where $\left|\mathcal{P}_{i}\right|$ is the volume of the ith particle. Let $\left|\mathcal{P}_{i}\right|(t+\delta t)=\left|\mathcal{P}_{i}(t)\right| \eta_{i}(t, \delta t)$ where $\eta_{i}$ is a correction factor. At each time step, the set of factors $\eta_{i}$ can be adjusted in order to enforce the previous condition. The corresponding set of linear equation can not be solved directly since it has a full matrix and the following iterative procedure has been used :

$$
\mathcal{G}\left(\underline{\mathbf{X}}_{i}, \underline{\mathbf{X}}_{j}, t\right)\left|\mathcal{P}_{i}(t+\delta t)\right| \eta_{i}(t, \delta t)^{n}=1-\sum_{j=1, I, i \neq j} \mathcal{G}\left(\underline{\mathbf{X}}_{i}, \underline{\mathbf{X}}_{j}, t\right)\left|\mathcal{P}_{j}(t+\delta t)\right| \eta_{i}(t, \delta t)^{n-1}
$$

It was found from numerical experiment that this method limits the error to the region close to the external boundary.

\section{Numerical tests and discussion}

\subsection{Diffusion in a turbulent jet}

The diffusion of a passive scalar in a turbulent jet is used as a test case for the three methods described above. This case has been selected because it involves transport by advection and diffusion by molecular and turbulent processes. To fairly compare the methods, the mixing length theory has been used to represent the effect of turbulence on the diffusion through a space-varying eddyviscosity coefficient. This approach provides differential relations for the jet quantities which can be numerically solved to determine velocity fields and eddy-viscosity fields. Here, two dimensional jets have been considered.

This computational case presents many of the difficulties that arise in particle methods :

- the shear rate in the jet is large so that distribution of particles in the jet is highly affected along time,

- external boundaries,

- the eddy-viscosity distribution has large variations in space.

Mean velocity fields and eddy-viscosity distribution are plotted in Figure (1).

The initial conditions are given by an exponential distribution for $c_{0}$, centred on point $(1.5,0)$. This distribution is plotted in Figure (2) together with the solution at time $t=0.5$ for a pure advection. 

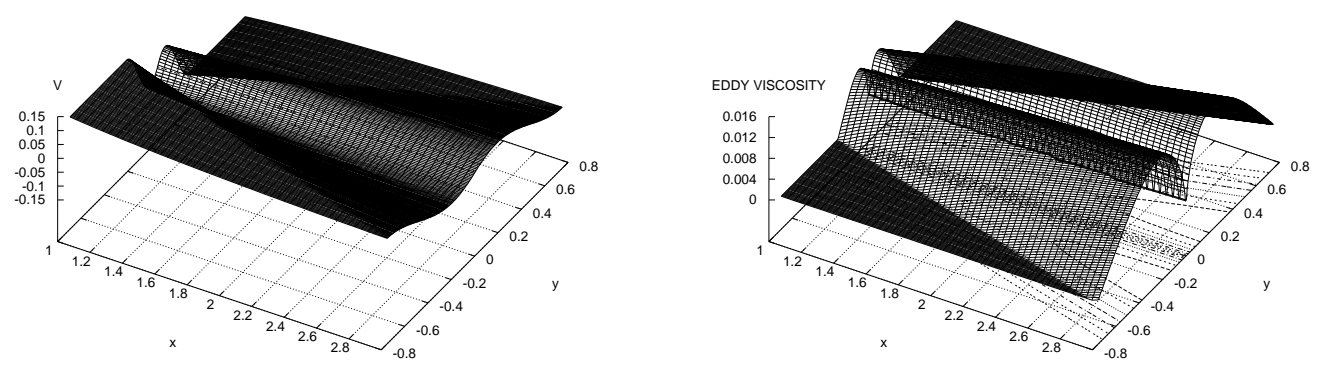

Figure 1: Vertical component of the mean velocity field and eddy-viscosity in the jet. Algebraic model based on the mixing length theory.
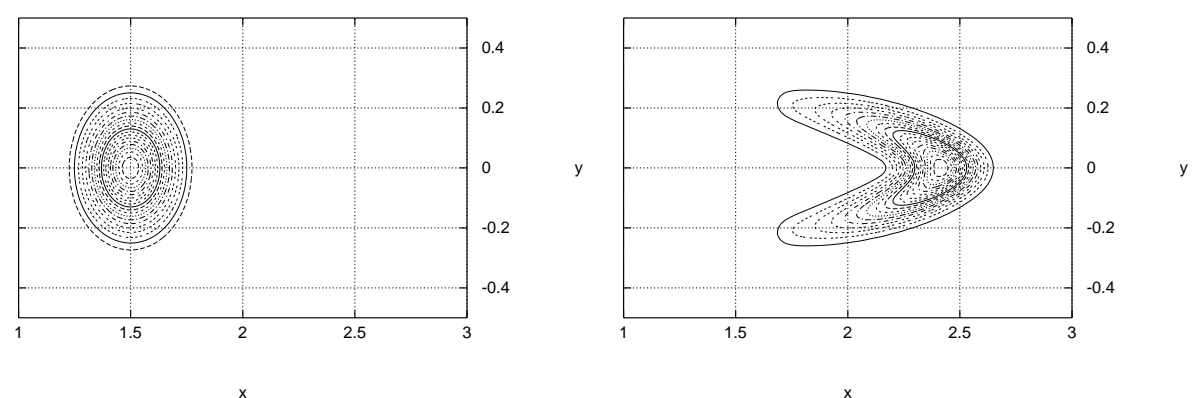

Figure 2: Initial concentration field for the passive scalar $c_{0}$ located in the jet core (left) and solution without any diffusion at time 0.5 (right). 

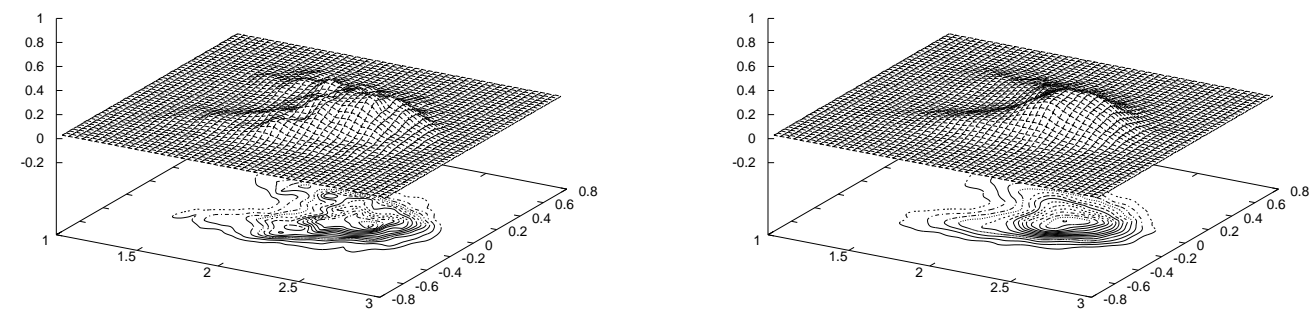

Figure 3: RVM : solution at time 0.5, with $\Delta t=0.01, \delta=0.01$, and using 2935 (left) or 9703 (right) particles .

\subsection{RVM results.}

The first results of diffusion with eddy-viscosity are presented for the RVM algorithm. Solutions are compared at time 0.5 for two initial discretizations of the scalar field : one using 2935 particles, ant the second 9703 particles. The time step is $\Delta t=0.01$ and $\delta=0.05$ in both cases. The results are plotted in Figure (3). It can be observed that the number of particles needed to obtain a smooth solution is large : for 2935 particles the solution is quite noisy. This is one of the drawback of the methods. It should be underlined that in your computations, the eddy viscosity is independent of the scalar field ( $\underline{\nabla} \nu$ is known) and the displacement of every particle is independent of the other leading to a low computational cost. Although, for turbulence modelling, the local value of the viscosity will depend on the solution and its evaluation will involve the full set of particles with large computational efforts as consequences. Moreover, for large times, the density of particles decreases at the external boundary of the perturbation with bad approximations as consequence.

\subsection{SEM results.}

Next the same test problem is solved using the SEM as presented above. It has been found that the SEM requires less particles than the RVM to compute smooth solutions. This is the advantage of a deterministic method on stochastic approach. Since the diffusion is model by weight exchanges between particles and because diffusion tends to enlarged the support of $c$, one has to extend the initial discretisation of the scalar field to allow for exchanges outside its initial support. To illustrate this difficulty and the error introduced by restricting diffusion to a bounded discretized domain, we have plotted in Figure (4) the solutions at $t=0.5$ for two discretisations : first, the initial distribution of particles only covers the support of $c_{0}$, and second, the initial distribution is enlarge of one diameter around the initial perturbation. The results clearly demonstrate the effects of limiting the domain covered by the particles : the solution becomes nearly uniform in the discretized domain with sharp variations at the boundaries. 

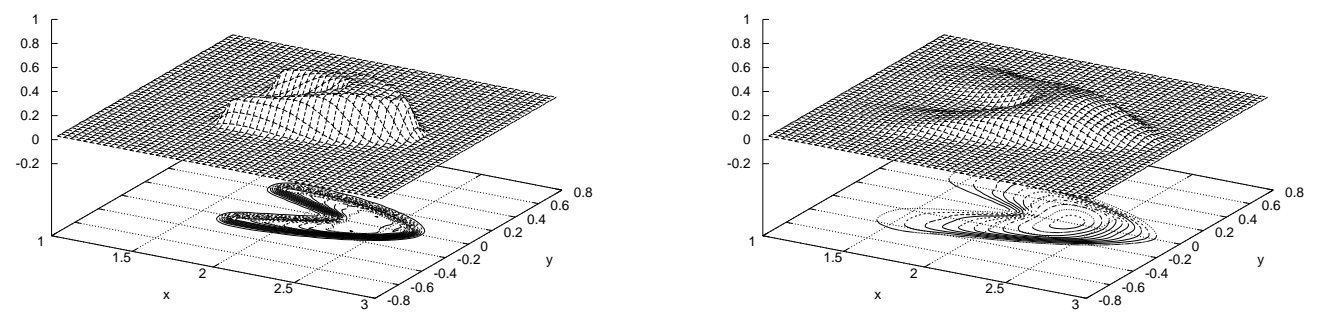

Figure 4: SEM : (left) solution at time 0.5 for $\Delta t=0.01$ and $\delta=0.01$. Left : using 1328 particles and an initial discretisation that only covers the support of $c_{0}$. Right : the initial discretisation is extended by one diameter and 2935 particles are used.
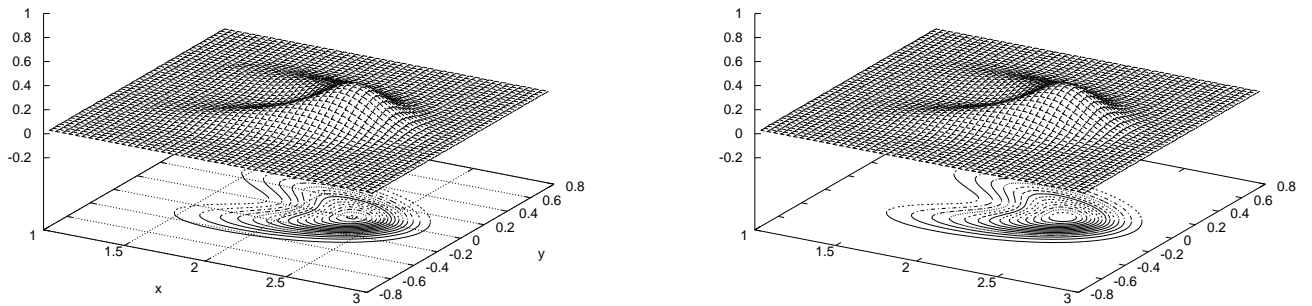

Figure 5: DVM : solution at time 0.5 , using 1328 particles. $\Delta t=0.01$ and $\delta=0.01$. The initial discretisation only covers the initial support of $c_{0}$. (Left) 1328 and (right) 2935 particles.

\subsection{DVM results.}

Finally, the DVM is tested on the previous problem. To compare the results with the SEM ones, the same numbers of particles have been used to discretize the initial field : 1328 and 2935 particles. In both case, the initial distribution of particles only covers the $c_{0}$ support, since the DVM is a constant weight algorithm. The results are plotted in Figure (5). It can be noticed that the two solutions are very similar : DVM can provide accurate results for a moderate number of particle and can naturally treat external boundary conditions at least for the simulated time presented here. For larger times, the density of particles near the boundary decreases and the approximation deteriorates. 


\section{Conclusion}

As a conclusion, it can be pointed out that all the methods are able to provide good results. The non uniformity of the diffusion coefficient does not affect too much the characteristics of the methods which are basically similar to the uniform case. The RVM requires a larger number of particles. This was not really a problem in our case since there was no interaction between the particles. The SEM is very sensitive to the external boundary conditions. As it has been mentioned, no regridding was used although some improvement can be expected from these techniques. The DVM seems to exhibit some advantages for this problem, but the effect of the space inhomogeneity on the interaction still have to be examined. It is clear that the computation of the diffusion coefficient and velocity fields from the information carried by the particles will lead to new problems. The particles number effect on the computing time as well as the possible loss of accuracy due to the increasing distance between particles for the RVM and DVM will have to be carefully examined before any significative conclusions could be drawn.

\section{References}

1 C. BREARD Etude des effets d'interaction fluide/ structure dans les écoulements instationnaires, Thèse de Doctorat de l'Université du Havre (1995)

2 J.P. Choquin , S. Huberson : Particles simulation of viscous flow Computers and Fluids. Vol $17 \mathrm{n}^{\circ}$ 2, pp.541-553, (1989).

3 A.J. Chorin : Numerical study of slightly viscous flows Journal of Fluid Mechanic, vol 57, pp 785-794, (1973).

4 G.H. Cотtet , S. Mas-Gallic A particle method to solve the Navier-Stokes system, Numer.Math. Vol 57, pp 1-23 (1990)

5 P. Degond , S. Mas-Gallic : The weighted particle method for convection diffusion equations -Part II : The anisotropic case

6 P. Degond , F.J. Mustieles : A deterministic approximation of diffusion equations using particles"SIAM J. Sci. Stat.Comput.Vol 11, pp 293-310, (1990).

7 D. Fischelov : A new vortex scheme for viscous flows, J. Comput. Phys. Vol 86 pp 211-224 (1990)

8 J. Fronteau , X. Combis : Hadronic Journal, vol 7, pp. 911 (1984).

9 A. Jolles : Résolution des équations de Navier Stokes par des méthodes particules maillage Thèse de Doctorat de l'Université Pierre et Marie Curie (1989)

$10 \mathrm{~S}$. MAS-GALLIC : Contribution à l'analyse numérique des méthodes particulaires Thèse d'Etat de l'Université Pierre et Marie Curie (1987)

11 .C. Mesneveau, J. Mansfeld and O. Knio : Towards lagrangian large vortex simulation , Vortex Flows and Related Numerical Methods II, ESAIM: Proceedings, Vol. 1, 1996, http://www.emath.fr/Maths/Proc/Vol.1

12 Y. Ogami , T. Akamatsu : Vicous flow simulation using a discrete vortex model Jour. of Computers and Fluid, Vol 19, pp. 433-441 (1991).

13 J. Sethian , A. Ghoniem : Validation study of vortex methods, J. Comput. Phys. Vol 54, pp 425-456 (1984).

14 J.H.Strickland, S.N. Kempka, W.P. Wolfe : Viscous Diffusion of Vorticity using the Diffusion Velocity Concept", Vortex Flows and Related Numerical Methods II, ESAIM: Proceedings, Vol. 1, 1996, http://www.emath.fr/Maths/Proc/Vol.1 